\title{
Notational conventions
}

\section{Manual signs}

SIGN

$\mathrm{IX}_{1}, \mathrm{IX}_{2}, \mathrm{IX}_{3}$

POSS $_{1}$, POSS $_{2}$, POSS $_{3}$

SIGN++
Signs are glossed with English words in small capitals $\mathrm{IX}$ is the abbreviation for index. The subscript numbers stand for locations in the signing space where a referent is located. $\mathrm{IX}_{1}=$ first person, $\mathrm{IX}_{2}=$ second person, $\mathrm{IX}_{3}=$ third person.

Possessive pronouns, the subscript numbers stand for locations in the signing space where a referent is located. Poss $_{1}$ $=$ first person, POSS $_{2}=$ second person, POSS $_{3}=$ third person Prosodic break

Crosses mark a reduplication of a sign or parts of a sign. Reduplication is used for different grammatical purposes such as the marking of plural. Inherent lexical reduplications are not marked in the glosses. 


\section{Nonmanual markings}

Nonmanuals are indicated by a line above the glosses for the manual signs. The length of the line represents the duration of the respective nonmanual marking. Lexical nonmanuals are not indicated in the glosses. Nonmanual markings relevant for the glosses in this book are listed below.

\begin{tabular}{lrl} 
& br-r & \\
\hline SIGN & SIGN & Brow raiser \\
& br-l & \\
\hline SIGN & SIGN & Brow lowerer \\
& h-n & \\
\hline SIGN & SIGN & Head nod \\
& h-s & \\
\hline SIGN & SIGN & Head shake \\
& h-f & \\
\hline SIGN & SIGN & Head forward \\
& h-d & \\
\hline SIGN & SIGN & Head down \\
& h-u & \\
\hline SIGN & SIGN & Head up
\end{tabular}

